\title{
Variabilité des soies spermathécales de Potamothrix heuscheri (Bretscher) (Oligochaeta, Tubificidae) dans le lac de Nemi et remarques sur la relation entre cette espèce et le degré de tro- phie des lacs
}

\author{
M. Bazzantit \\ M. Lafont ${ }^{2}$
}

Mots clés : Oligochaeta, Variations morphologiques, Eaux stagnantes, Ecologie, Degré de trophie.

Les auteurs rapportent ici quelques données sur la variabilité des soies spermathécales constatée chez des exemplaires du Tubificidae Potamothrix heuscheri (Bretscher), récoltés dans le lac de Nemi (Italie Centrale). L'étude systématique, basée sur l'anatomie de l'appareil efférent mâle et sur la morphologie des soies génitales permet de conclure qu'il existe un écotype de l'espece dans ce lac.

La distribution de $P$. heuscheri dans les lacs français et italiens, en particulier dans le lac de Nemi, est discutée dans ce travail pour tenter de clarifier le problème posé par les exigences écologiques de cette espèce, actuellement peu connues et parfois contradictoires, en milieu lacustre.

Variability in the sperma thecal setae of Potamothrix heuscheri (Bretscher) (Oligochaeta, Tubificidae) in Lake Neml and notes on the relationship between this species and the trophic level of lakes

Keywords : Oligochaeta, Morphological variations, Standing waters, Ecology, Trophic level.

Data are presented on variability in the spermathecal setae of the tubificid Potamothrix heuscheri (Bretscher) from Lake Nemi (Central Italy). A systematic study, based on the antomy of the efferent apparatus of the male and the morphology of the genital setae, indicates that there is an ecotype of this species in this lake.

The distribution of $P$. heuscheri in French lakes, especially Lake Nemi, is discussed in order to clarify the problem posed by the ecological requirements of this species in lakes because these requirements are little known and sometimes contradictory.

\section{Introduction}

En milieu lacustre, les Oligochètes benthiques constituent en général de bons indicateurs du degré de trophie d'un plan d'eau, du fait qu'ils vivent en contact étroit avec le sédiment et que leur développement s'effectue exclusivement en milieu aquatique. Par ailleurs, lorsque les peuplements d'Oligochètes, en particulier les Tubificidae, se rencontrent en proportion élevee dans la faune benthique, on considère qu'il y a un état d'emrichissement en matières organiques de l'eau dans laquelle ils vivent

\footnotetext{
1. Dipartimento di Biologia Animale e dell'Uomo, Università * La Sapienza ". Viale dell'Università 32, 00185, Roma, Italia.

2. Laboratoire d'Hydrobiologie du CEMAGREF, 3 quai Chau veau, 69009 Lyon, France.
}

(Goodnight \& Whitley 1961, Brinkhurst \& Jamieson 1971, Howmiller \& Beeton 1971, Aston 1973). Cependant, au sein de la famille des Tubificidae, les espèces réagissent différemment aux facteurs de l'environnement et présentent des distributions différentes dans les milieux dont les degrés de trophie sont variables. Plusieurs auteurs (Howmiller \& Scott 1977, Lang \& Lang-Dobler 1980, Wiederholm 1980, Milbrink 1980, 1983) ont établi, dans le but d'évaluer le degré d'eutrophisation d'un lac, des indices basés sur l'abondance de diverses especes indicatrices d'Oligochètes.

Alors que pour plusieurs espèces, la tolérance à la pollution organique et aux conditions d'anoxie qui en résultent est bien connue dans la littérature, il n'en va pas de même pour le Tubifícidae Potamothrix 
heuscheri, essentiellement à cause de sa relative. ment récente apparition en Europe centrale et du nord (Milbrink 1980). Il nous a donc paru intéressant, afin de contribuer à mieux connaître l'écologie de cette espèce, de rapporter quelques observations sur cet Oligochète, récolté en pourcentages élevés dans un lac fortement eutrophe, le lac de Neni.

Le lac de Nemi est un petit lac volcanique d'une superficie de $1,670 \mathrm{~km}^{2}$ et dont la profondeur maximale atteint environ 31 mètres. Dans la dernière décennie, les eaux de tout le plan d'eau ont reçu des effluents d'origine donestique qui ont entrainé une eutrophisation rapide et forte du lac. Cette accumulation de substances a causé depuis 1974 des altérations chiniques et biologiques qui ont déjà fait l'objet de divers travaux (Stella \& al., 1978 ; Bazzanti \& Loret, 1982 ; Bazzanti, 1983 ; Ferrero \& Gelosi, sous presse). La concentration élevée en sels dissous (P total $50 \mu \mathrm{g}^{-1}$ ), la désoxygénation de la zone profonde plusieurs mois par an, la présence $\mathrm{d}^{\prime} \mathrm{H}_{2} \mathrm{~S}$ au fond et les graves perturbations biologiques, (proliférations de Cyanophycées, fortes mortalités de poissons, etc...) mettent clairement en évidence le stade avancé de dégradation des eaux du lac. Par ailleurs, dans ce même lac, des exemplaire de Potamothrix, présumés appartenir à l'espèce heuscheri, présentaient des variations sensibles dans la forme des soies spermathécales, nécessitant un examen systématique approfondi, qui va constituer la première partie de ce travail.

\section{1. - Soies spermathécales de Pota- mothrix heuscheri dans le lac de Nemi}

Le Tubificidae Potamothrix heuscheri fut décrit pour la première fois en 1900 par Bretscher (in Brinkhurst 1971) sous le nom de Tubifex heuscheri. Les variations pouvant exister dans la forme des soies génitales n'ont pas, à notre connaissance, fait l'objet de mentions détaillées dans la littérature. En général, les auteurs (Piguet et Bretscher 1913, Cekanovskaya 1962, Hrabe 1954, 1981, Kasprzak 1981. Pop 1976) représentent des soies spermathécales appartenant à des types sensiblement identiques (cf. fig. $1 \mathrm{~A}$ ). L'examen de 114 individus sexuellement matures, récoltés dans le lac de Nemi, et présumés appartenir à l'espèce $P$. heuscheri par référence à la forme des soies copulatrices, a permis de mettre en évidence des variations dans la forme de ces soies. Celle-ci semblait s'écarter du type communć. ment décrit dans la littérature. La question s'est alors posée de savoir si nous n'étions pas en pré. sence d'une espèce nouvelle.


Fig. 1. Soies spermathécales de $P$. heuscheri;A : selon Pop, 1976 ; B : mesures effectuées $; \mathrm{D}=$ longueur de la dent la plus longue $: \mathbf{H}+\mathbf{N}=$ longueur de la hampe + le nodulus.

\subsection{Matériel et méthodes.}

Les exemplaires fixés au formol ont été montés entre lame et lamelle dans du liquide de Faure ou dans un mélange à parts égales d'acide lactique et de glycérine. Une douzaine de spécimens porteurs ou non de soies génitales aberrantes furent disséqués et montés dans du sirop de lévulose. Les soies spermathécales ont été mesurées selon les modalités suivantes : le contour externe de chaque soie est tracé sur une feuille de papier à l'aide d'un microscope muni d'une chambre claire et mesuré au moven d'un curvimetre préalablement étalonné. Deux mesures ont été effectuées (fig. 1B) : longueur de la dent la plus longue (D) et longueur de la hampe + le nodulus $(\mathbf{H}+\mathbf{N})$. De ces deux mesures, la longueur totale de la soie $(\mathrm{L})$ est déduite $: \mathrm{L}=\mathrm{D}+\mathbf{( H}$ $+\mathrm{N}$ ). Cette procédure implique l'élimination de 


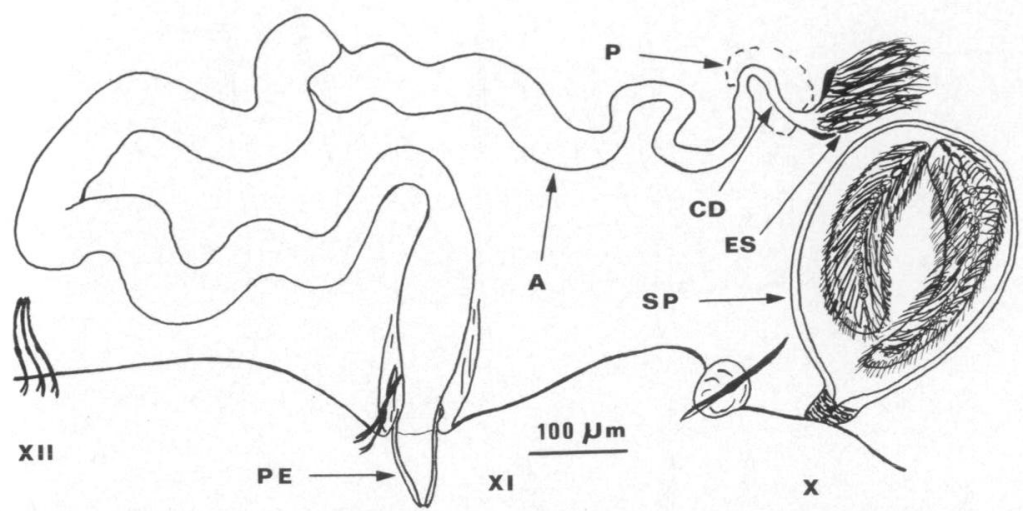

Fig. 2. Reconstruction semi-schématique d'après dissections de l'appareil efférent mâle de $P$. heuscheri du lac de Nemi ; ES : entonnoir séminal ; $C D$ canal déférent ; $P$ : prostate ; $A=$ atrium ; $P E$; pénis ; $\mathbf{S P}$ : spermathèque; les métamères sont notés en chiffres romains.

toutes les préparations où les soies spermathécales sont déformées, cassées et/ou non montées dans un plan horizontal : 83 soies seulement (sur 228 ) ont pu faire l'objet de mesures correctes.

\section{2. - Résultats}

\subsubsection{Appareil efférent mâle, (fig. 2)}

Il se compose d'un entonnoir séminal, d'un canal déférent très court auquel fait suite un atrium long et glandulaire. Celui-ci est contourné, les boucles qu'il forme débordant largement dan's le segment XII. Cet atrium porte un renflement avant de déboucher au pore $\sigma$ (métamère XI). Le pénis, musculeux, n'est apparemment pas protégé par une gaine cuticularisée, au contraire de $P$. hammoniensis (Baker 1982). Les spermathèques possèdent une ampoule globuleuse et des conduits très courts. L'anatomie de l'appareil efférent $\sigma$ de nos exemplaires du lac de Nemi ne s'écarte guère de celle que Piguet \& Bretscher (1913) ou Brinkhurst (1971) ont décrite.

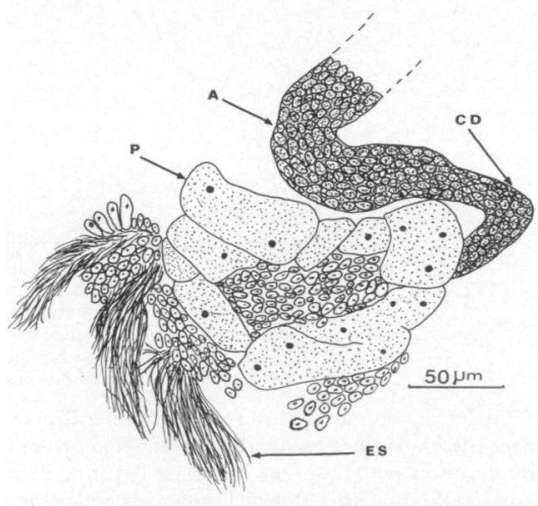

Fig. 3. Croquis, d'après dissection, de l'extrémité antérieure de l'appareil efférent mâle de $P$. heuscheri (Lac de Nemi). ES sentonnoir séminal ; P : prostate ; A : atrium : $\mathrm{CD}$ :canal déférent. 

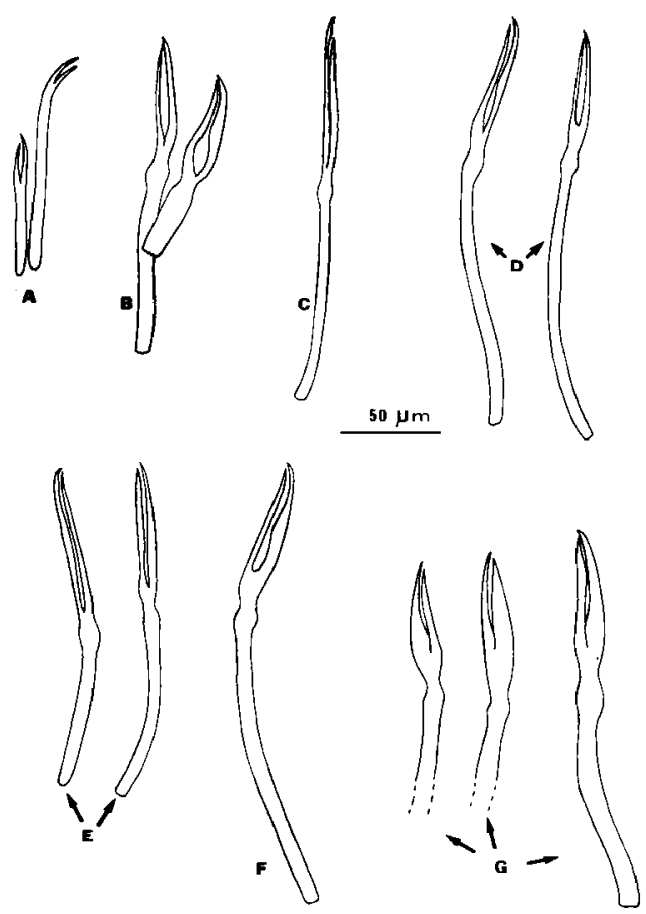

Fig.4. Soies copulatrices des exemplaires de P. heuscheri du lac de Nemi ; A : en cours de formation ; $B$ : faisceau du $10^{\mathrm{e}}$ segment renfermant une soie bien formée typique de l'espèce, et une soie à dent élargie en cours de formation (dents formées, hampe incompletement formée); $\mathbf{C}$ : soie typique de l'espece ; $\mathbf{D}$ : soies à hampe allongée ; $\mathbf{E}$ : soies à hampe courte ; $\mathbf{F}$ : soies a dents élargies, avec une hampe longue ; $\mathrm{G}$ : soies a dents élargies avec une hampe de longueur moyenne.

Ce dernier auteur, à l'inverse de Piguet \& Bretscher (1913) signale l'absence de prostate. Nous avons pu toutefois remarquer, au départ de l'atrium, des amas cellulaires (fig. 3) dont l'aspect rappelle très sensiblement celui d'une prostate de Tubificidae. Juget (comm. pers.) a observé des structures analogues sur des exemplaires disséqués de $P$. heuscheri en provenance d'un bras mort du Rhóne.

\subsubsection{Description des soies spermathécales}

On sait que les soies génitales, spermathécales ou péniennes, ont pour origine des soies somatiques ventrales. Sur nos exemplaires du lac de Nemi, cette genèse a pu être en partie observée. Les dents des crochets somatiques s'allongent (fig. 4A), puis la hampe s'allonge à son tour lorsque les dents sont 
bien formées (fig. $4 \mathrm{~B}$ ). Les différences observées par rapport à des soies considérées comme typiques de $P$. heuscheri (fig. 4C) portent essentiellement sur la forme des dents ou sur l'allongement plus ou moins accentué de la hampe. En effet, $18 \%$ des soies ont des dents de forme épaissie rappelant celle des soies de Potamothrix bedoti (fig. 4B, 4F, 4G) les autres sont typiques de l'espèce $P$. heuscheri (ftg. 4C, 4D, 4E), mais dans les deux cas, les hampes des soies sont longues (fig. 4D, 4F), courtes (fig. 4B, 4E) ou de taille normale (fig. $4 \mathrm{C}$ et $4 \mathrm{G}$ ). Les mesures effectuées sur les soies montrent qu'il existe une corrélation linéaire hautement significative $(r=0,95)$ entre la longueur de la hampe + le nodulus $(\mathrm{H}+\mathrm{N})$ d'une part, et la longueur totale de la soie (L) d'autre part. La corrélation linéaire entre la longueur de la dent la plus longue (D) et $\mathrm{L}$ est plus faible $(r=0,58)$. Enfin, il n'existe pas de corrélation linéaire entre $\mathrm{D}$ et $\mathrm{H}+\mathrm{N}$. On remarque également que dans un mêrne faisceau ( $f$ ig. 4B), il peut $y$ avoir coexistence des deux types de crochets spermathécaux.

Il ne semble donc pas faire de doute que la longueur des hampes est une conséquence de l'âge des soies, les soies « âgées " ayant des hampes longues, les soies " jeunes " des hampes courtes, que ce soit dans le cas des soies à dent élargie ou non. En dernier lieu, l'étude de la distribution des valeurs du rapport $m=\frac{H+N}{D}$ montre que $9 \%$ des soies mestrées présentent un rapport $\mathrm{m} \leqslant 1,1$ (= soies " jeunes $n) ; 24,8 \%$ des soies mesurées ont un rapport $m$ $\geqslant 1,8$ (soies " âgées ". Pour la plupart des crochets génitaux (67\%), le rapport $m$ est compris entre 1,2 et 1,7. Parmi ces dernières valeurs se situent également celles qui ont été obtenues à partir de mesures, effectués à l'aide d'un curvimètre, sur des crochets reproduits dans l'iconographie de la littérature (op. cit.) ou sur des exemplaires de P. heuscheri de diverses provenances (notamment le lac Léman, la Saulx, le-Rhône, le Doubs).

Pour les exemplaires du lac de Nemi, la longueur totale des soies copulatrices varie de $110_{\mu}$ m à 240 ${ }_{\mu} \mathrm{m}($ moyenne $=171 \mu \mathrm{m})$.

\section{3. - Conclusion.}

L'anatomie de l'appareil efférent mâle et la taille de la hampe des soies spermathécales ne permettent pas de conclure à l'existence d'une espèce nouvelle dans le lac de Nemi. Il faut noter également que la mesure des soies est un critère délicat à interpréter, compte-tenu des nombreux artéfacts pouvant survenir lors de la fixation et du montage des spécimens de vers entre lame et lamelle (soies brisées, déformées, montées verticalement...). En revanche, il est très probable que les exemplaires où se rencontrent des soies à dents élargies (fig. 4B, 4F, 4G) appartiennent à un écotype de l'espèce $P$. heuscheri. Ce phénomène a été signalé chez les Naididae (Loden \& Harman 1980) ou d'autres organismes vivants (Harman 1980). La coexistence, dans le même milieu, de l'archétype et de son ou ses écotypes est un phénomène connu en milieu lacustre (Juget 1967 b). Nos observations sur la variabilité des soies génitales de $P$. heuscheri amèneraient des arguments supplémentaires en faveur de l'hypothèse de Wautier et Juget (1969) sur le rôle inducteur des facteurs écologiques dans la spéciation des peuplements benthiques lacustres.

\section{2. - Distribution de Potamothrix heuscheri dans des plans d'eau présentant des degrés de trophie différents}

$P$. heuscheri, originaire de la région pontocaspienne (Milbrink 1980, Timm 1980), a été signalé dans les eaux douces de plusieurs pays d'Europe (Brinkhurst 1978) et dans des eaux marines côtières (Laasko 1969, Milbrink 1980). Dans le lac Léman, les exemplaires appartenant à Potamothrix heus. cheri ont été récoltés aussi bien dans des zones mésotrophes (Lang \& Lang-Dobler 1979, 1980) qu'eutrophes (Lang 1978, Lang \& Hutter 1981). Martinez-Ansemil \& Prat (1984) observent cette espèce dans quelques lacs de barrage espagnols caractérisés par des eaux alcalines et oligotrophes. Milbrink $(1973,1978,1980,1981)$ signale que P. heuscheri est susceptible de vivre dans des milieux très productifs et eutrophes; ce même auteur a trouvé cette espèce dans les eaux polluées du bassin nordest du lac Mälaren, et place $P$. heuscheri dans un groupe de Tubificidae tolérant des conditions eutrophes ou une pollution organique considérable. En outre, il est particulièrement intéressant de noter la présence de ce Tubificidae, formant des populations monospécifiques, dans la zone profonde privée 
d'oxygène du lac saumâtre de Tibériade (Gitay 1968), où il peut supporter de longues périodes d'anoxie ( 8 mois) et des concentrations élevées en hydrogène sulfuré. Dans des conditions expérimentales, cette même espèce peut survivre six mois au maximum en l'absence d'oxygène (Por \& Masry 1968).

En France, $P$. heuscheri a été en général peu mentionné jusqu'ici, que ce soit dans les cours d'eau (Giani 1979, Lafont 1983, Juget \& Roux 1982) ou dans les lacs. Ce tubificidae n'est connu pour l'instant que dans le lac Léman de 8 à $120 \mathrm{~m}$ de profondeur où il peut être abondant (Juget 1967 a), dans le lac du Bourget (Michel 1966) à $145 \mathrm{~m}$ de profondeur où il ne représente que moins de $2 \%$ des peuplements d'Oligochètes, dans quelques lacs de barrage du sudest de la France (Grégoire 1982), et dans le lac de Petichet (Alpes françaises, $923 \mathrm{~m}$ d'altitude, Gay, 1976). Ce dernier plan d'eau se caractérise par des déficits en $\mathrm{O}_{2}$ dissous $\left(0,58 \mathrm{mgl}^{-1}\right.$ à $-19 \mathrm{~m}$ au mois de septembre), mais surtout par des concentrations élevées en $\mathrm{NH}_{4}{ }^{+}\left(2,71 \mathrm{mgl}^{-1}\right)$ et en $\mathbf{H}_{2} \mathrm{~S}\left(3 \mathrm{mgl}^{-1}\right)$ à la fin de l'été dans les eaux profondes $(-19 \mathrm{~m})$. P. heuscheri forme alors des populations monospécifiques relativement peu abondantes en moyenne (1621 indivi. dus $/ \mathrm{m}^{2}$ ). Gay (1976) considérait que la répartition verticale de cette espèce semblait être en relation avec l'augmentation de la charge organique des sédiments (avec la profondeur). Juget (1984) obtient d'ailleurs une relation similaire dans un sédiment d'un bras mort du fleuve Rhône.

Dans les lacs italiens ${ }^{3}, P$. heuscheri est assez répandue et se récolte en Italie du nord dans la zone littorale du lac majeur (Nocentini 1963), dans le lac d'Iseo (Bonomi \& Gerletti 1967) le lac de Garde (Bonomi 1974), le lac d'Endine (Nocentini \& al.1974), dans le bassin ouest du lac d'Annone (Istituto Italionao di idrobiologia, Pallanza 1978), en Italie centrale, dans les lacs de Monterosi (Stella et Margaritora 1966, Bazzanti el al sous presse), de Martignano (Stella et al., 1972), de Vico (Bonomi \& Ruggiu 1971, Nocentini 1973), de Bracciano (Bonomi \& Ruggiu 1971, Nocentini 1973, Bazzanti 1981), de Nemi (Bazzanti \& Loret 1982, Bazzanti 1983), et de Canterno (Bazzanti \& Seminara 1984).

3. Actuellement, nous ne possédons que peu de données sur les communautés d'Oligochètes des lacs de l'Italie méridionale, où cependant la présence de $P$. heuscheri n'a pas été signalée.

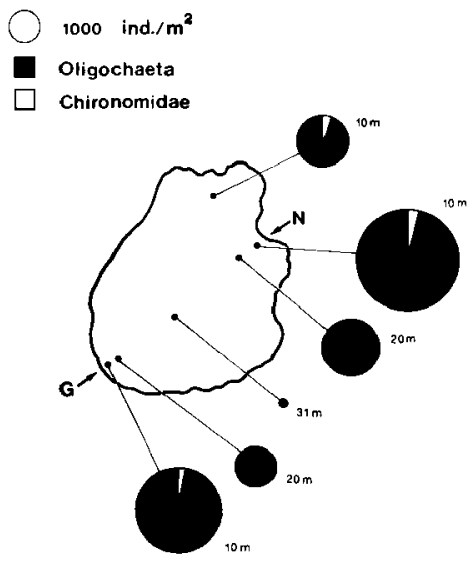

Fig. 5. Abondance des deux groupes dominants, Oligochètes et Chironomidae, dans le lac de $\mathrm{Nemi}$; $\rightarrow$ indique la zone de déversement des effluents domestiques provenant des villages de Nemi (N) et Genzano (G).

Selon les données bibliographies (op. cit.), P. heuscheri se distribue aussi bien dans des eaux oligotrophes et mésotrophes (par exemple les lacs de Garde, de Martignano et de Bracciano) que fortement eutrophes ou polluées (lacs d'Endine, d'Annone, de Canterno et de Nemi). Bien que constituant une proportion appréciable des peuplements d'Oligochètes, $P$. heuscheri ne se révèle actuellement pas, dans les plans d'eau concernés, comme une espèce fortement dominante parmi les peuplements de macroinvertébrés benthiques, à l'exception du lac de Nemi.

\section{1. - Distribution de Potamothrix heuscheri dans le lac de Nemi}

La faune benthique profonde (fig. 5) du lac de Nemi est composée presque exclusivement d'Oligochètes qui représentent plus de $97 \%$ de la faune du fond ${ }^{4}$.

4. Ces valeurs numériques se réfèrent a des données récoltées au cours des années d'étude du lac (1979-1980). La méthodologie utilisée et les stations de recolte sont décrites dans le travail de Bazzanti (1983) 


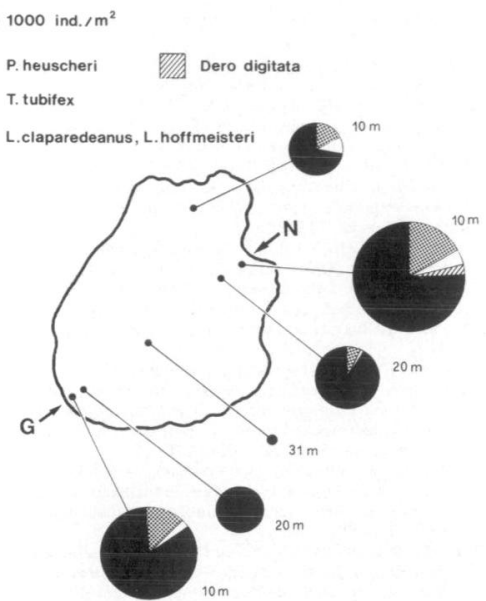

Fig. 6. Distribution et abondance des espèces d'Oligochètes dans le lac de Nemi.

$P$. heuscheri constitue l'espèce la plus répandue du plan d'eau ; elle est associée à Tubifex tubifex, Limnodrilus hoffmeisteri, L. claparedeanus et Dero digitata, espèce récoltée en faibles quantités à 10 mètres de profondeur seulement (fig. 6). A $-10 \mathrm{~m}$ de profondeur, $P$. heuscheri présente sa densité absolue la plus élevée et forme 72,1 à $86,6 \%$ des peuplements d'Oligochètes et 68,9 à $84,5 \%$ de la macrofaune benthique. A - $20 \mathrm{~m}$ de profondeur, l'abondance absolue de $P$. heuscheri diminue, mais son abondance relative augmente $(91,7$ à $100 \%$ des Oligochètes, 91,6 à 100 $\%$ de toute la faune benthique). A la profondeur maximale $(-31 \mathrm{~m})$, le macrobenthos n'est plus représenté que par $P$. heuscheri qui forme des populations peu abondantes ( 100 individus $/ \mathrm{m}^{2}$ ).

\section{2. - Discussion}

D'après les données chimiques et biologiques recueillies dans le lac de Nemi, il apparait clairement que $P$. heuscheri peut survivre dans des conditions de forte pollution organique. Les plus fortes densités de ce Tubificidae se remarquent surtout dans les zones directement soumises aux rejets d'origine domestique (effluents des villages de Nemi et Genzano). Par ailleurs, $P$. heuscheri se trouve associée à d'autres espèces de Tubificidae telles que $T$. tubifex, $L$. hoffmeisteri et $L$. claparedeanus, dont la tolérance à la pollution organique est bien connue (Brinkhurst 1965, Aston, 1973 ; Brinkhurst et Cook 1974, Milbrink 1980, Chapman \& al. 1982 a). La présence de $P$. heuscheri dans des lacs de niveaux trophiques différents n'a rien de surprenant, un tel phénomène est assez connu et a été observé également pour d'autres espèces qui présentent une valence écologique plus ou moins large, comme T. tubifex et $S$. ferox (Lang \& Lang-Dobler 1979, 1980, Milbrink $1980,1983)$. Comme l'a souligné Lafont $(1977,1982)$, la polluo-résistance d'une espèce est un caractère autécologique relatif, étroitement lié aux conditions ambiantes (type de substrat, compétition, type de pollution etc...). Pour évaluer le degré d'eutrophisation d'un plan d'eau, il est donc nécessaire de connaître d'une manière approfondie, outre les paramètres abiotiques, la composition des communautés d'invertébrés benthiques, en tenant compte soit de la proportion d'une espèce au sein d'un groupe, soit de la composition qualitative et quantitative des communautés d'invertébrés benthiques dans toute leur complexité.

Dans le cas du lac de Nemi, les pourcentages élevés de $P$. heuscheri, ainsi que sa présence en populations monospécifiques dans la zone la plus profonde du lac, sembleraient démontrer que les capacités d'adaptation de ce Tubificidae à des conditions extrêmes sont supérieures à celles de $T$. tubifex et de $L$. hoffmeisteri. Cette situation pourrait être attribuable à deux phénomènes concomitants :

a) lorsque des espèces d'Oligochètes entrent en compétition avec d'autres, la densité de leur population se réduit. Actuellement, les modalités de distribution sympatrique des espèces d'Oligochètes ne sont pas encore bien claires, mais il semble que plusieurs facteurs interviennent dans sa régulation (Brinkhurst \& al. 1972, Juget 1979, Lang \& Lang-Dobler 1979, Milbrink, 1980, Särkkä \& Aho 1980, Lang \& Hutter 1981, Särkkä 1982) ;

b) des travaux récents (Chapman \& al., 1982 b) ont montré en particulier que certaines espèces de Tubificidae, lorsqu'elles sont ensembles, sont moins 
tolérantes à des conditions d'anoxie qu'une espèce seule. Ceci pourrait expliquer la présence de populations monospécifiques dans des milieux où règnent des conditions de totale absence d'oxygène.

Des recherches ultérieures, conduites soit au laboratoire, soit sur le terrain, avec des eaux de différentes qualités, permettront d'éclairer les exigences écologiques du Tubificidae $P$. heuscheri, et les facteurs agissant sur la régulation de ses populations, afin d'obtenir, avec plus de précision, la place de cette espèce dans une échelle de niveaux trophiques, valable aussi bien pour les eaux italiennes que françaises.

\section{Remerciements}

Les auteurs remercient Monsieur J. Juget qui a bien voulu accepter de critiquer ce manuscrit, Madame Taillole qui a assuré la dactylographie du texte et Monsieur Balland qui a calculé les coefficients de régression.

\section{Travaux cités}

Aston (R.J.). 1973. - Tubificids and water quality : a review. Environ. Poll, $5: 1-10$.

Baker (H.R.). 1982, - A note on the genitalia of Potamothrix hammoxiensis (Oligochaeta, Tubificidae). Proc. biol soc. Wash., 95 (3) : 563-566

Bazzanti (M.). 1981. - Survey of the macrobenthic community in an area of Lake Bracciano (Central Italy). Boll. Zool, 48 (3-4) : 295-303

Bazzanti (M.). 1983. - Composition and diversity of the profundal macrobenthic community in the polluted Lake Nemi (Cen. tral Italy), 1979.1980. Acta Oecologica, Oecol. Applic., 4 (3) : 211-220.

Bazzanti (M.), Ferrara (O.) et Mastrantuono (L.). (sous presse). Osservazioni sul le attuali condizioni del lago-stagno di Monterosi (Lazio). Boll. Pesca. Piscic. Idrobiol.

Bazzanti (M.) \& Loret (E.). 1982. - Macrobenthic community structure in a polluted lake : Lake Nerni. Boll. Zool, 49 (1-2) : 79-91.

Bazzanti (M.) \& Seminara (M.). 1984. - Macrobenthos estivo ed autunale di un lago inquinado soggetto a variazioni di livello dell' acqua. Boll. Zool, 51, suppl. : 8

Bonomi (G.). 1974. - Benton profondo. - In : Indagini sul Lago di Garda. Quaderni IRSA, $18: 211-223$.

Bonomi (G.) \& Gerletti (M.). 1967. - Il lago d'Iseo : primo quadro limnologico generale (termica, chimica, plancton e benton profondo). Mem. Ist. Ital. Idrobiol., 22 : 149-175.

Bonomi (G.) \& Ruggie (D.) 1971 - Popolamenti bentonici pro fondi. In : Limnologia ed ecologia dei laghi di Bolsena, Brac. ciano, Trasimeno e Vico: situazione attuale e prevedibili con seguenze derivanti da una loro utilizzazione multipla. Rd. Ist $\mathrm{i}$ tuto Italiano di Idrobiologia, Pallanza : 227-248.

Brinkhurst (R.O.). 1965. - Observations on the recovery of a british river from gross organic pollution. Hydrobiologia, 25 (1-2) 9.51.
Brinkhurst (R.O.). 1971. - Tubificidae. - In : Aquatic Otigochaeta of the World. Brinkhurst R.O. et Jamieson BG.M. eds., Oliver and Boyd, Edinburh: $\mathbf{4 4 4 6 2 5}$.

Brinkhurs1 (R.O.) 1974. - The Benthos of Lakes. The Mac Millan Press Ltd, $190 \mathrm{pp}$.

Brinkhurst (R.O.). 1978. - Oligochaeta. - In : Limnofauna Euro paea. Illies (J.) ed., A. Fisher Verlag, Stuttgart: 139-144

Brinkhurst (R.O.), Chua (K.E.), \& Kaushik (N.) 1972. - Interspecific interactions ans selective feeding by tubificid oligochaetes. Limnol. Oceanogr., $17:$ 122-133.

Brinkhurst (R.O.) \& Cook (D.G.). 1974, - Aquatic earthworms (Annelida : Oligochaeta). - In : Pollutiom ecology of freshwater invertebrates. Hart C.W.Jr et Fuller S.L.H. eds., Academic Press, London : 143-156.

Cekanovskaya (O.V.) 1962, - The aquatic Oligochaete fauna of the U.S.S.R. Opred. Faune S.S.S.R., 78:1-411.

Chapman (P.M.), Farrell (M.A.) \& Brinkhurst (R.O.) 1982 a. Relative toierances of selected aquatic oligochaetes to individual pollutants and environmental factors. Aquat. Toxic, 2 : 47.67 .

Chapman (P.M.), Farrell (M.A.) \& Brinkhurst (R.O.). 1982 b. Effects of species interactions on the survival and respiration of Limnodrilus hoffmeisteri and Tubifex tubifex (Oligochaeta, Tubificidae) exposed to va rious pollutants and environmental factors. Water Res., 16: 1405.1408.

Ferrero (L.) \& Gelosi (E.). (sous presse). - Studio dell'inquinamento di un lago vulcanico laziale : il Lago di Nemi. Consequenze dell' alterazione dell' ambiente sulla produzione ittica. Boll. Pesca Piscic. Idrobiol.

Gay (C.) 1976. - Contribution à l'étude écologique et à l'aménagement d'un lac de montagne: le lac de Petichet (lsère), (altitude : $923 \mathrm{~m}$ ). Thèse de Doctorat de Spécialité, Grenoble : 124 Pp.

Giani (N.). 1979. - Les Oligochetes aquatiques du Sud-Ouest de la France (deuxième note), Bull. Soc. Hist. Nal. Toulouse, 115 (3-4) : 347-358.

Gitay (A.). 1968. - Preliminary data on the ecology of the levelbottom fauna of Lake Tiberias. Isr. J. Zool., 17:81-96.

Goodnight (C.J.) \& Whitley (L.S.). 1961. - Oligochaetes as indicators of pollution. Proc. 15th Amn. Waste Conf., Purdue Univ. : 139-142.

Grégoire (A.). 1982. - Contribution à l'étude hydrobiologique d'une rivière aménagée: le Verdon. Les lacs de barrage et les tronçons de cours d'eau à débit régulè. Cah. Lab. Hydr. Montereau. $13: 1-162$.

Harman (W.J.) 1980. - Specific and generic criteria in freshwater Oligochaeta, with special emphasis on the Naididae. In : Aquatic Oligochaete biology. Brinkhurst R.O. et Cook D.G. éds., Plenum Press, New-York and London : 1-8.

Howmiller (R.P.) \& Beeton (A.M.). 1971. - Biological evaluation of environmental quality. Green Bay, Lake Michigan. $J$. Wat. Pollut. Control Fed. 43 (1): 123-133

Howmiller (R.P.) \& Scott (M.A.) 1977. - An environmental index based on relative abundance of Oligochaete species. J. Wat. Pollut. Control Fed., 49 : 809-815.

Hrabe (S.). 1954. - Malostetinatci - Oligochaeta Klic zvireny CSR, $1: 289-323$.

Hrabe (S.). 1981. - Vodni malostetinatci (Oligochaeta), Ceskoslovenska. Acta Universilatis Carolinae, Biologica, 1979 (1-2): 1-168.

Is tituto Italiano di Idrobiologia Pallanza (1978) - Lago di Annone. Regione Lombardia, $148 \mathrm{pp}$.

Juget (J.). 1967 a. - La faune benthique du Lèman : modalités et déterminisme écologiques du peuplement. Thèse de Doctorat d'Etat, Lyon: $360 \mathrm{pp}$.

Juget (J.). 1967 b. - Quelques données nouvelles sur les Oligochètes du Léman : composition et origine du peuplement. Annls Limnol., 3 (2): 217-229. 
Juget (J.). 1979, - La texture granulométrique des sédiments et le régime alimentaire des Oligochètes limicoles. Hydrobiolo gia, $65(2): 145-154$

Juget (J.). 1984. - Oligochaeta of the epigean and underground fauna of the alluvial plain of the french upper Rhóne (biotypological trial), Hydrobiologia, $115:$ 175-182.

Juget (J.) \& Roux (A.L.). 1982, - Une lone du Rhóne, zone humide en position de lisière dans l'espace et dans le temps. Bull Ecol, 13 (2): 109.124

Kasprzak (K.). 1981. - Skaposzczety wodne I; klucze do oznak. zania bezkregowcow Polski. Polska Akad. Nauk, IV : 1-221.

Laakso (M.). 1969. - Oligochaeta from brackish water near Tvằminne, South-West Finland, Arn. Zool. Fenn., 6: 98-111.

Lafont (M.). 1977. - Les Oligochètes d'un cours d'eau montagnard pollué : le Bief Rouge. Annts. Limnol, 13 (2) : 157-167.

Lafont (M.). 1982. - Les Oligochètes des lacs de Gerardmer et de Longemer (département des Vosges, France). Sciences de l'Eau, L (1) : 21.30

Lafont (M.). 1983 - Les Oligochètes. In : Etude hydrobiologique de la Saulx Marnaise après aménagement d'une partie de son cours. Rapport CEMAGREF Lyon: 26-35

Lang (C.). 1978. - Approche multivariable de la détection biologique et chimique des pollutions dans le Lac Léman (Suisse). Arch. Hydrobiol., 83 (2) : 158.178.

Lang (C.) \& Hutter (P.) 1981 - Structure, diversity and stability of two Oligochaete communities according to sedimentary inputs in Lake Geneva (Switzerland). Schweiz. Z. Hydrol, 43 (2): $265 \cdot 276$.

Lang (C.) \& Lang-Dobler (B.). 1979. - The chemical environement of tubificid and lumbriculid worms according to the pollution level of the sediment. Hydrobiologia, 65 (3): 273-282.

Lang (C.) \& Lang-Dobler (B.). 1980. - Structure of tubificid and lumbriculid worm communities, and three indices of trophy based upon these communities, as descriptors of eutrophication level of Lake Geneva (Switzerland). In : Brinkhurst R.O., Cook D.G., eds., Aquatic Oligochaete biology. Plenum Press, New-York and London : 457.470.

Loden (M.S.) \& Harman (W.J.). 1980. - Ecophenotypic variation in setae of Naididae (Oligochaeta). In : Aquatic Oligochaete biology, Brinkhurst R.O., Cook D.G. éds., Plenum Press, New.York and London : 33-39.

Martinez-Ansemil (E.) \& Prat (N.). 1984. - Oligochaeta from profundal zones of Spanish reservoirs. Hydrabiologia, 115 : 223-230.

Michel (S.). 1966. - Recherches sur la faune benthique du lac du Bourget. D.E.S., Lyon : $106 \mathrm{pp}$.

Milbrink (G.) 1973. - On the use of indicator communities of Tubificidae and some Lumbriculidae in the assessment of water pollution in Swedish lakes. Zoon, 1:125.139

Milbrink (G. ). 1978. - Indicator communities of Oligochaetes in Scandinavian lakes. Verh. Intemat. Verein. Limmol., 20 : 2406-2411.
Milbrink (G.). 1980. - Oligochaete communities in pollution bio logy : the european situation with special reference to lakes in Scandinavia. In : Brinkhurst R.O., Cook D.G., eds. Aquatic Oligochaete biology. Plenum Press, New-York and London : 433-455.

Milbrink (G.). 1983. - An improved environmental index based on the relative abundance of Oligochaete species. Hydrobiologia, $102: 89-97$

Nocentini (A.M.). 1963. - Strutture differenziali della fauna macrobentonica litorale del Lago Maggiore. Mem. Ist. Itol. Idrobiol, 16 : 189.274

Nocentini (A.M.). 1973. - La fauna macrobentonica litorale e sublitorale dei laghi di Bolsena, Bracciano e Vico(Italia Centrale, Lazio). Mem. Ist. Ital. Idrobiol., $30: 97-148$.

Nocentini (A.M.), Ruggiu (D.) \& Saraceni (C.). 1974. Popolamento bentonico. In ; Indagini ecologiche sul Lago di Endine. Istituto Italiano di Idrobiologia, Pallanza ed, : $260-288$.

Piguet (E.) \& Bretscher (K.). 1913. - Oligochètes. In : Catalogue des Invertébrés de la Suisse. Mus. Hist. Nat Genève, $7: 1-214$.

Pop (V.). 1976. - Ist Potamothrix thermalis (Pop) synonym mit Potamothrix heuscheri (Bretscher) (Tubificidae, Oligochaeta, Annelida)? Zool. AMz lena. 196 (3-4): 196-200.

Por (F.D.)) \& Masry (D.). 1968. - Survival of a nematode and Oligochaete species in the anaerobic benthal of Lake Tiberias. Oikos, 19 (2) : 388-391.

Saether (O.A.). 1980. - The influence of eutrophication on deep lake benthic invertebrate communities. Prog. Wat. Tech.. 12: 161-180.

Särkä (J.). 1982. - On the ecology of littoral Oligochaeta of an Oligotrophic finnish lake. Holarciic Ecology, 5 (4) : 396-404.

Särkkä (J.) \& Aho (J.). 1980. -- Distribution of aquatic Oligochaeta in the Finnish Lake Distric. Freshwal. Biol., 10 : 197-206.

Stella (E.), Ferrero (L.) \& Margaritora (F.G.). 1978. - Alterations of the plankton in a much polluted lake in central Italy (Latium), the volcanic Lake Nemi. Verh. In. Verein. Limnol., 20: 1049.1054

Stella (E.) \& Margaritora (F.G.). 1966. - Studio faunistico ed ecologico di un lago-stagno del lazio : il Lago di Monterosi. Archo Zool. Ital., $51: 159.226$

Stella (E.), Margaritora (F.G.), Palmegiano (G.B.) \& Bazzanti (M.). 1972. - Il Lago di Martignano: prime osservazioni sulla struttura e distribuzione delle biocenosi. Acad. Naz, $X L, 22$ : $1-17$

Timm (T). 1980 - Distribution of aquatic Oligochaetes. In : Brinkhurst R.O. and Cook D.G., eds., Aquatic Otigochaete bio lagy. Plenum Press. New-York and London : 55-77.

Wautier (J.) et Juget (J.). 1969. - La faune berthique et la spéciation dans les lacs subalpins. Bull Soc. Zool. France, 94 (2): 277.284

Wiederholm (T.). 1980. - Use of benthos in lake moniloring. $J$. Wat. Pollut. Control Fred., 52 (3) : 537-547. 